\title{
LTE-U and Wi-Fi Hidden Terminal Problem: How Serious Is It for Deployment Consideration?
}

\author{
Anand M. Baswade*, Touheed Anwar Atif ${ }^{\dagger}$, Bheemarjuna Reddy Tamma*, Antony Franklin A* \\ * Indian Institute of Technology Hyderabad, India. \\ ${ }^{\dagger}$ University of Michigan, Ann Arbor, USA. \\ Email: [cs14resch11002*, tbr*, antony.franklin*]@iith.ac.in, touheed ${ }^{\dagger} @$ umich.edu
}

\begin{abstract}
The deployment of LTE in unlicensed spectrum is a plausible solution to meet explosive traffic demand from mobile users. However, fair coexistence with the existing unlicensed technologies, mainly Wi-Fi, needs to be ensured before any such deployment. Duty cycled LTE (LTE-U) is a simple and an easily adaptable scheme which helps in fair coexistence with the Wi-Fi. Nonetheless, the immense deployment of $\mathrm{Wi}-\mathrm{Fi}$ necessitates a user-oriented study to find the effects of LTE-U operation, primarily in scenarios where the LTE-U eNB remains hidden from Wi-Fi Access Point. To comprehend these effects, we perform a user-level throughput study of $\mathrm{Wi}-\mathrm{Fi}$ in the presence of LTE-U using a testbed and observe a clear unfairness in throughput distribution among Wi-Fi users. Furthermore, we also notice inability among the disadvantaged users to receive the periodic Wi-Fi beacon frames successfully. The reasons and the subsequent consequences, of throughput unfairness and beacon losses, are carefully elaborated. Also, to validate the beacon loss results, we present a beacon loss analysis which provides a mathematical expression to find the beacon loss percentage. Finally, we examine the results and highlight a need for incorporating additional functionalities in either LTE-U or Wi-Fi to overcome the present challenges.
\end{abstract}

\section{INTRODUCTION}

The last few years have seen a rapid increase in cellular data traffic demand [1], [2] due to a sudden surge in the usage of smart phones and tablets. To manage such high user data demands, the telecom industry is keen on utilizing the unlicensed spectrum [3], [4]. Although LTE in unlicensed might fulfill these demands, along with improving the spectral efficiency of unlicensed spectrum - it must ensure fair coexistence with other technologies in unlicensed spectrum, mainly IEEE 802.11 (a.k.a. Wi-Fi)—before being widely accepted. Some of the approaches like Licensed Assisted Access (LAA) which follows Listen Before Talk (LBT) [3], a similar channel access mechanism like $\mathrm{Wi}-\mathrm{Fi}$; and duty cycled discontinuous transmission, with LTE eNB following an ON-OFF cycle pattern (called as LTE-U) [4]-[8]; claims to fairly coexist with Wi-Fi. However, discontinuous and duty cycled transmission approach, due to its simplicity of requiring minimal changes in the existing LTE protocol, is being pushed to the markets. One such example is Carrier Sense Adaptive Transmission (CSAT) [9], where eNB follows an ON-OFF cycle pattern, with $\mathrm{ON}$ and OFF durations corresponding to LTE transmissions and muting duration, respectively.

Furthermore, [10] shows both LTE-U CSAT scheme and LBT to be equally fair with $\mathrm{Wi}-\mathrm{Fi}$ and leaves it on the operator
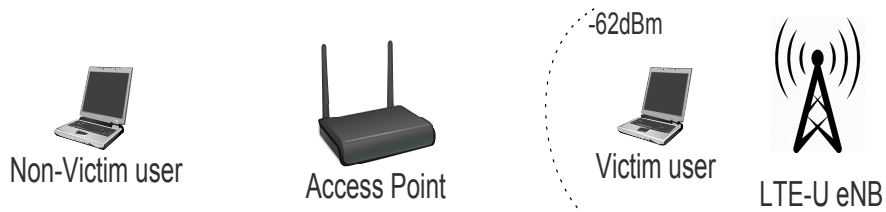

Fig. 1. Scenario shows a Wi-Fi AP with two users and an LTE-U eNB with both the networks operating on the same unlicensed channel. The victim Wi-Fi user is inside the influence zone of LTE-U; and the non-victim Wi-Fi user along with Wi-Fi AP are outside the influence zone of LTE-U.

to decide which scheme to deploy. But, due to the current ubiquitous deployment of $\mathrm{Wi}-\mathrm{Fi}$, there are scenarios where very intricate challenges can prevail. We consider one such class of scenarios and delineate thoroughly the complications involved in achieving fair throughput distribution among the Wi-Fi users and the difficulties arrived in attaining consistent beacon reception by these $\mathrm{Wi}-\mathrm{Fi}$ users in such scenarios.

The class of scenarios which we consider is in fact, very similar to the $\mathrm{Wi}-\mathrm{Fi}$ hidden terminal problem, with LTE-U eNB required to be hidden from the Wi-Fi AP while the AP may or may not be hidden from the LTE-U eNB . The scenario essentially consists of a Wi-Fi network partially overlapped with the LTE-U network as shown in Fig. 1; with the Wi-Fi AP outside the influence zone of LTE-U, and thus can transmit or receive data even during the LTE-U ON period. We define the influence zone of LTE-U as the region around LTE-U eNB where a Wi-Fi device cannot transmit or receive successfully when LTE-U is ON because of the following two reasons. Firstly, the signal strength received by the Wi-Fi device from the LTE-U transmissions is high enough compared to the Clear Channel Assessment (CCA) Threshold [11] of the Wi-Fi device, causing it to sense the channel busy and halt from any transmission. Secondly, the interference caused by the LTE-U signals to the Wi-Fi device is substantial and thereby decreases the Signal-to-Interference-plus-Noise-Ratio (SINR) below the minimum SINR required for successful reception. Now, we consider some of the $\mathrm{Wi}-\mathrm{Fi}$ users to be present within the influence region of LTE-U and call them as victim users. Consequently, the remaining $\mathrm{Wi}-\mathrm{Fi}$ users which are outside the influence region are called as non-victim users.

The main contributions of the paper can be summarized as follows:

- We analyze the considered hidden terminal scenario on a testbed setup, and study the performance of Wi-Fi users 


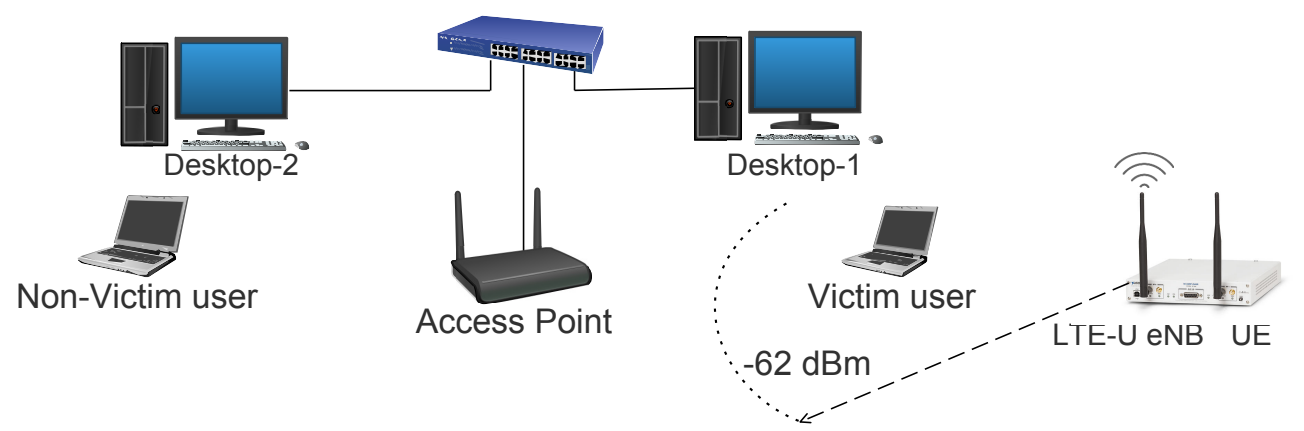

Fig. 2. Experimental testbed setup demonstrating the Wi-Fi network partially overlapped with the LTE-U network, with additional two Desktops used for sending and receiving iPerf traffic to/from the Wi-Fi users.

in the presence of duty cycled LTE-U.

- We observe the unfairness caused to the victim users in terms of throughput and also study the effect of the presence of these victim users on a Wi-Fi network. The lack of comprehensive literature for such scenarios using real hardware makes our study novel.

- We study the beacon lost phenomena of victim users and present the effects of beacon losses. We also propose beacon loss analysis and provide a mathematical expression to calculate the beacon loss percentage. Finally, we validate the analytical results using simulation and the testbed.

The rest of the paper is organized as follows. In Section II, the related work is discussed. Experimental setup and results are shown in Sections III and IV, respectively. In Section V, beacon loss analysis for victim users is proposed. Finally, conclusions and future work are given in Section VI.

\section{RELATED WORK}

Though the telecom industry is very keen to make LTE to operate in unlicensed spectrum, the research community is concerned about LTE-U fairly sharing the spectrum with other unlicensed technologies. Hence, most of the work in the literature focuses on the fair coexistence of LTE-U/LAA with Wi-Fi [12], [13]. The foremost claim made is that the deployment of LTE in unlicensed without changing LTE protocol will significantly degrade the performance of Wi-Fi. This was shown in [14] using a system-level simulator. In [15], the performance of LTE and Wi-Fi in a shared frequency band was presented which again showed that LTE degrades the performance of $\mathrm{Wi}-\mathrm{Fi}$, but to improve the performance of Wi-Fi a muting technique was introduced within LTE, while maintaining fairly good performance of LTE.

In [16], the authors apart from evaluating through simulations the impact on performance of Wi-Fi by LTE when both operate in the same frequency, suggested a modified almost blank subframe approach in LTE for fair coexistence with Wi-Fi. In fact, most of the work in literature is focused on simulation and/or mathematical modeling. On the other hand, in [17] the performance degradation of Wi-Fi was studied using a testbed when a traditional LTE network operates in the same unlicensed channel. However, a Wi-Fi user oriented performance study using testbed is unprecedented. In this paper, we focus on the class of scenarios where the effect of LTE is dissimilar for different set of Wi-Fi users. Furthermore, to make our study more realistic we consider a more justifiable LTE operation enabled with discontinuous duty cycled transmissions, and study the effect on Wi-Fi users using testbed. We also propose a beacon loss analysis for the so called victim users and validated the results using the testbed.

\section{Experimental Testbed Setup}

The experimental testbed setup consists of an LTE-U network partially overlapped with a Wi-Fi network-with one user as victim and another as non-victim-as shown in Fig. 2. The center frequency for LTE-U and Wi-Fi is set to $2.442 \mathrm{GHz}$ (i.e., Wi-Fi channel 7).

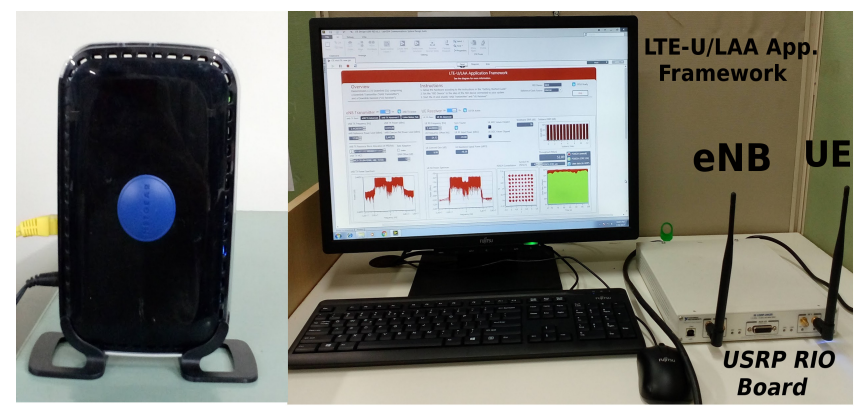

Fig. 3. Equipment used in the testbed: (i) Netgear N600 wireless dual band router WNDR3400v3 used as Wi-Fi AP. (ii) USRP RIO board with LTE-U eNB and LTE-U user operated using LTE-U/LAA Application framework.

In [18], National Instruments (NI) demonstrated a realtime LTE-U and Wi-Fi coexistence testbed and provided an application framework for LTE-U/LAA. This framework was developed by modifying the existing NI LTE application framework [19]. We have employed the USRP RIO board with the same NI LTE-U/LAA application framework [18] to create an LTE-U eNB and an LTE-U user. Furthermore, a Wi-Fi network is setup using a commercial "Netgear N600 wireless dual band router WNDR3400v3" as an AP. The equipments used for Wi-Fi AP and LTE-U eNB are shown 
in Fig. 3. The Wi-Fi AP is operating in 802.11n mode with two other Laptops (Ubuntu 14.04 LTS with Intel wireless 8260 chipset and Realtek drivers) as two Wi-Fi stations. The two Laptops along with two Desktops are used for a client-server application each installed with iPerf [20], with Desktops being connected to the switch using $1 \mathrm{~Gb} / \mathrm{s}$ Ethernet cables as shown in Fig. 2. For the traffic flow in the network, we configured LTE-U eNB to be transmitting only in downlink (as LTE in unlicensed is used only in downlink [3], [4]) and studied Wi-Fi performance for the following two scenarios. First, Downlink (DL) only, containing solely the DL traffic. Second, Uplink (UL) and DL containing both UL and DL traffic. To direct the DL traffic of the Wi-Fi network to its stations for both the scenarios, we configured the Wi-Fi network in infrastructure mode with AP as the primary entity, responsible for all DL transmissions. UDP is used to generate traffic for each flow in both the networks which makes it easier to distinguish between the UL and DL traffic.

For coexistence of LTE-U with Wi-Fi, the LTE-U follows a $\mathrm{ON}$ and OFF patterns, where it transmits for few milliseconds during the ON period and becomes silent in the OFF period. The experiments are performed to study the throughputs and beacon loss percentage of Wi-Fi users with different LTE-U ON periods. This variation in $\mathrm{ON}$ period is achieved by identically varying the ON-OFF periods in such a way that the complete duty cycle period (LTE ON + LTE-U OFF) is always $10 \mathrm{~ms}$. Each experiment is performed several times in order to remove the undesirable randomness and understand the average behavior of the network.

\section{EXPERIMENTAL RESULTS}

The performance of the deployed Wi-Fi stations is observed while varying the LTE-U ON fraction where, LTE-U ON fraction is a fraction of time LTE-U is ON in a given duty cycle period. As discussed earlier, the two main issues are highlighted-degradation in throughput and losses in beacon reception.

\section{A. Throughput Results}

For the throughput measurement of Wi-Fi network, a clientserver application named iPerf [20] is installed and is used for generating UDP traffic at a rate of $10 \mathrm{Mb} / \mathrm{s}$. The throughput performance of Wi-Fi stations is measured for both DL only and UL+DL traffic scenarios.

1) DL only scenario: In the DL only scenario, client applications are set up in the Desktops which transmit UDP packets to the servers listening at the victim and non-victim users, via the Wi-Fi AP. Throughput calculations are made after every iteration, with each iteration running for 40 seconds, by varying the LTE-U ON period. Furthermore, the same experiment is performed with two different packet sizes to observe the effect of packet size on the performance of Wi-Fi users, mainly the victim user. In addition, the performance of non-victim user, by muting the victim user, is also shown to highlight the effect of the presence of victim user on the non-victim user and the Wi-Fi network.

Following observations can be made from the experiments:

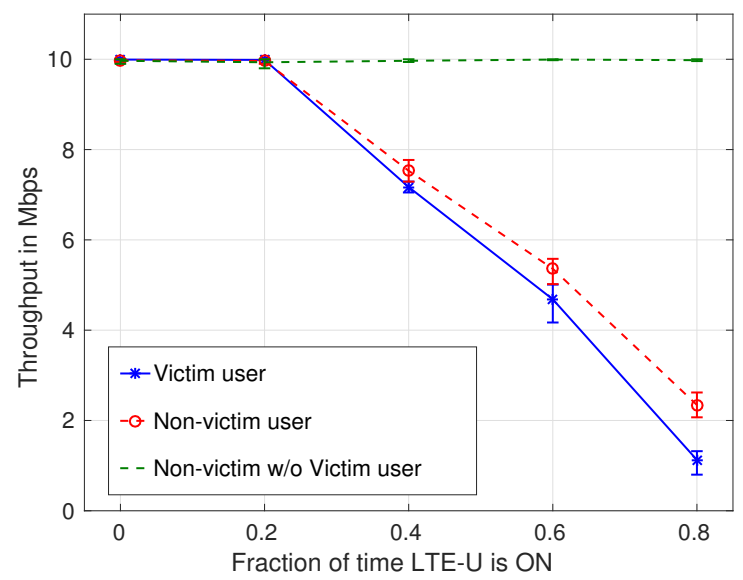

Fig. 4. Throughput of victim and non-victim users with varying LTE-U ON fraction and also the throughput of non-victim user without the presence of victim user for UDP datagram size of $200 \mathrm{~B}$.

a) Substantial retransmission losses leading to a decrease in throughput of victim as well the non-victim users: From Figs. 4 and 5, it can be observed that the performance of non-victim user, with victim user being muted, is independent of LTE-U ON period and remains at the maximum possible throughput of $10 \mathrm{Mb} / \mathrm{s}$. However, with the introduction of victim user traffic, not just the achievable throughput of victim descends with increasing LTE-U ON period but also the presence of victim user wrenches the throughput of nonvictim user. The decrease in the throughput of victim user with ON period is quite anticipated-with the decrease in duration where victim user can receive packets successfully the throughput of victim user is expected to decrease. However, the decrease in non-victim users throughput is unexpected and can be explained as follows. During the LTE-U ON period, the transmissions to non-victim user would be successful, but the transmissions to the victim, due to high interference, would result in a packet loss. Wi-Fi AP regards this transmission to be a collision and re-transmits the same packet, but by exponentially incrementing its Contention Window $(\mathrm{CW})$ and probably selecting a higher Back-Off (BO) value. This leads to a profuse increase in the total retransmissions in the Wi-Fi network. As the non-victim user is also served by the same AP, which is all but wasting most of the ON period doing retransmissions, the non-victim user also gets starved and eventually receives a decreased throughput.

b) Disproportionate throughput distribution among victim and non-victim users: Distributed Coordination Function (DCF) of Wi-Fi ensures equal throughput distribution among its users which can be seen from Figs. 4 and 5 at low LTE-U $\mathrm{ON}$ fraction. But as the $\mathrm{ON}$ fraction increases, an unbalance is created among the throughput distribution to victim and nonvictim users. This is because victim users can receive packets only in the LTE-U OFF period, whereas non-victim users can receive packets both in the LTE-U ON and OFF periods. In principle, LTE-U ceases to access the channel, during its OFF 


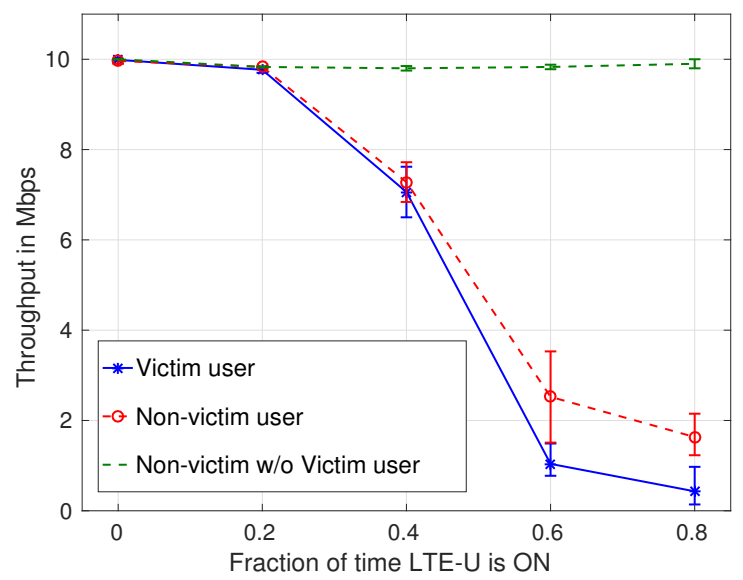

Fig. 5. Throughput of victim and non-victim users with varying LTE-U ON fraction and also the throughput of non-victim user without the presence of victim user for UDP datagram size of $1500 \mathrm{~B}$.

cycle, so that the victim users get channel access and thereby achieve a proportionate share of the channel and hence the throughput. Non-victim users access to the channel-during this (LTE-U OFF) period-engenders lower throughput to these victim users. This results in unfairness among Wi-Fi users which further increases with the LTE-U ON period.

c) Restriction on the packet size meant for victim users: The final observation which can be made from Figs. 4 and 5 is the dependence of packet size on victim users throughput. Although higher packet sizes give higher throughputs, but if the size of packet meant for victim user is considerably large, such that the air time of the packet exceeds the LTE-U OFF period, the packet would merely be lost. Moreover, if the packet air time is less but still significant compared to the OFF period, the probability that the packet would occur at the transition from an OFF to ON period would be high and would again result in a packet loss.

In addition, since the Wi-Fi AP was unable to serve the victim users during the LTE-U ON period, after receiving an opportunity in the LTE-U OFF period, it tries serving these victim users with minimal rates (due to the rate control algorithm), consequently increasing the packet air-time by multitudes. This unnecessary increase in the air-time and the limited OFF period, restricts the packet size and eventually becomes a compromise with the throughput. From the above figures, a comparable performance among victim and nonvictim users can be observed with 200B (in Fig. 4) and 1500B (in Fig. 5) packet sizes for low ON periods, but as ON period ascends the throughput of transmissions involving 1500B packets noticeably descends and reaches very low.

2) $U L+D L$ scenario: For a UL + DL scenario, the client and server applications (using iPerf) are setup in the Desktops as well as in the Laptops, so as to transmit packets in both UL and DL, via the Wi-Fi AP. Throughput measurements are made while varying the LTE-U ON period, with each flow having a rate of $10 \mathrm{Mb} / \mathrm{s}$ and a UDP datagram size of $200 \mathrm{~B}$.

Key observations for the UL + DL scenario are

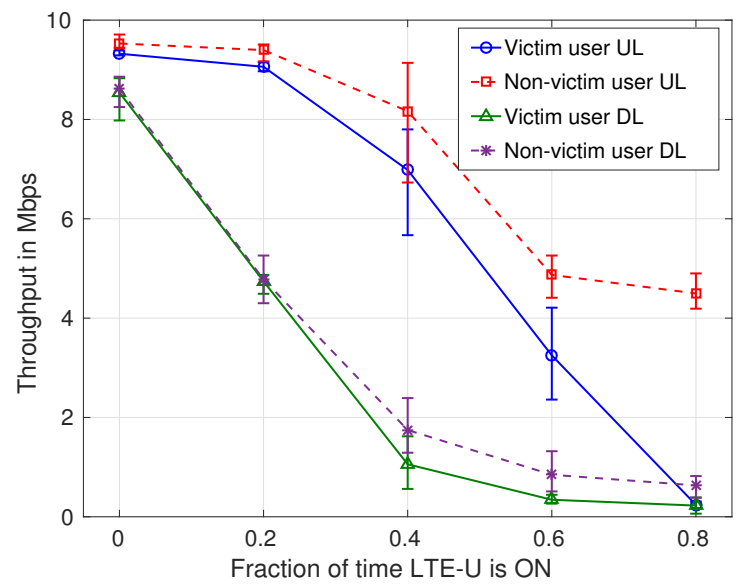

Fig. 6. Throughput of victim and non-victim users with varying LTE-U ON fraction in UL and DL.

a) Preferential Uplink transmissions over Downlink transmissions: Fig. 6 shows the variation in UL and DL throughputs of victim and non-victim users, with increasing LTE-U ON fraction. It can be seen that the UL throughputs of both the users are greater than their DL throughputs. As discussed earlier, the Wi-Fi AP being completely unaware of the fact that the packets meant to victim users are lost because of ongoing LTE-U ON period, tries re-transmitting packets to the victim users. Since every retransmission escalates the $\mathrm{CW}$ exponentially, multiple retransmissions would mean a sudden inflation in average $\mathrm{BO}$ value of the Wi-Fi AP, thus reducing the channel access ability of the AP. This results in a decrease in the DL throughputs of both the victim and non-victim users. While on the other hand, the non-victim users being distanced from the effect of LTE-U, gain an advantage to the channel in comparison with Wi-Fi AP. This can lead to a considerable increase in UL throughput in contrast to DL throughput.

b) Decrease in UL throughput for all users with increasing LTE-U ON period: From Fig. 6, the decrease in UL throughput of victim user is quite expected-with increase in LTE-U ON fraction, the channel available for the victim user to contend and transmit data decreases, and thereby reduces its throughput. However, a decrease in non-victim users UL throughput is astonishing. The fact to be perceived here is that the LTE-U transmissions not just affect the victim users but also decrease the UL SINR of the non-victim users (though not less than the minimum required). This causes the non-victim users to choose lower Modulation and Coding Schemes and consequently decrease their throughput.

c) A proportional effect on DL throughput for all users: From Fig. 6, it can be seen that the effect of LTE-U on DL throughputs is commensurate for both the victim and non-victim user. Although Wi-Fi AP can communicate with the non-victim user during the LTE-U ON period, the AP's convention of performing re-transmissions to the victim user, leaves the AP with very less $\mathrm{ON}$ period in which it can 
successfully transmit to non-victim and provide a perquisite over victim user. Therefore, with the increase of LTE-U ON time, the DL throughputs of all the users decreases.

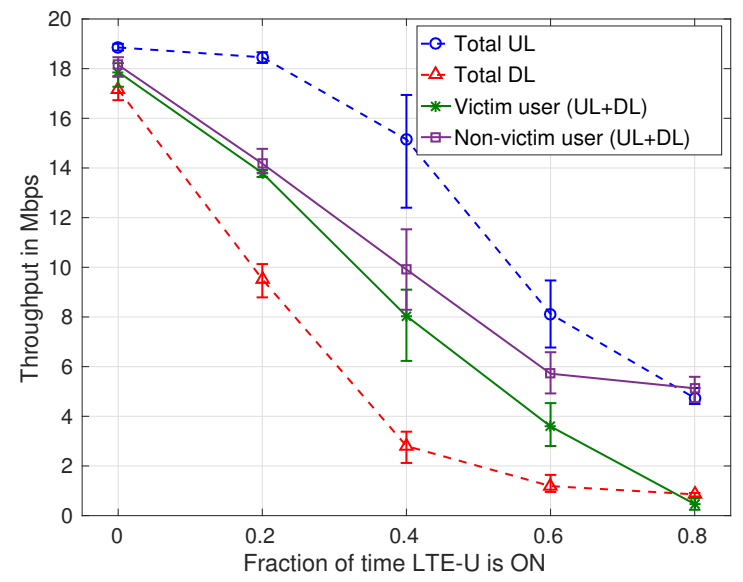

Fig. 7. Total UL and DL throughputs and total victim and non-victim users throughputs with varying LTE-U ON fraction.

Fig. 7 shows the total throughput of Wi-Fi network in UL and DL as well as the individual total throughput of victim and non-victim users with varying LTE-U ON fraction. Again the total DL throughput is lower than the total UL throughput. As the individual DL throughputs are less than the individual UL throughputs, it is certain that the total DL throughput of the network would be less than the total UL throughput of the network. In addition, the unfairness in the performance among the victim and non-victim users can also be observed. Moreover, this unfairness further increases with increasing LTE-U ON fraction.

Although the hidden terminal class of scenarios are discussed above in the perspective of duty cycled LTE-U scheme, the impact on Wi-Fi victim users holds true even for the case of LAA. The LBT mechanism of LAA may reduce the overall impact on the Wi-Fi network, but unfairness among users would still remain unjustified.

\section{B. Beacon Loss Results}

According to the IEEE 802.11 standard, APs are typically configured to periodically send out beacon frames. The purpose of a beacon frame is to advertise the presence of an AP; its capabilities; encryption protocol being used and also flags meant for the stations which convey them the information on presence of any packets in the buffer to be transmitted in the upcoming beacon interval-an essential feature for station operating in power saving mode.

Although beacon reception is indeed very crucial, LTE-U transmissions in the scenarios presented above can engender many beacons to be lost in-succession by the victim users. For evaluating the average beacon loss percentage, we used the same setup described in Section III. With the help of Wireshark (an open source Wi-Fi packet analyzer) [21], we captured the beacon frames on the victim user's laptop. Using the time stamp of the beacon frame provided by Wireshark, we were able to identify the number of missed beacons between two successfully received beacons. Consequently, the fraction of losses were calculated using these missed beacons and then averaged over many iterations for different LTE-U ON fractions. Fig. 8 shows one such instance for LTE-U ON fractions of $0.2,0.4,0.6$. As the beacon interval was 102.4 milliseconds, the presence of a peak at an interval of every $102.4 \mathrm{~ms}$ indicates a successful beacon reception, and the absence denotes a beacon loss. It can be seen from the same figure that the beacon losses increase with increasing LTE-U ON fraction.
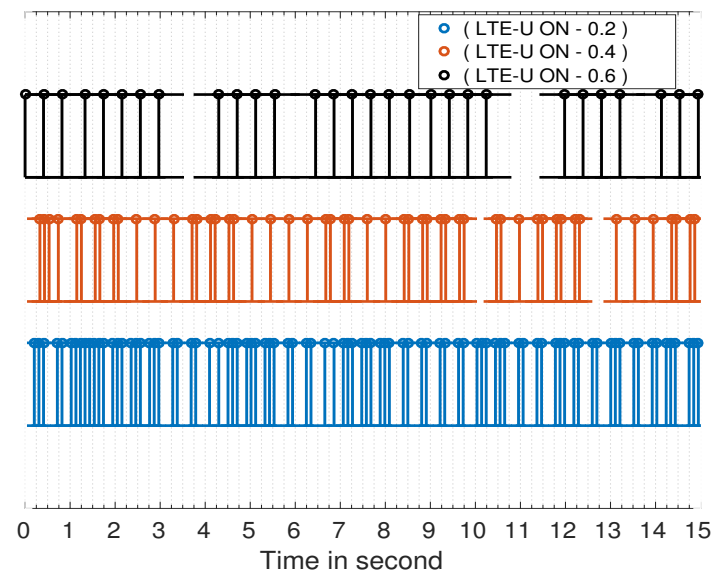

Fig. 8. Beacons received over time for different LTE-U ON-OFF fractions.

Furthermore, Table I shows a significant observation regarding the percentage of continuous beacon losses. The reason behind these consecutive beacon losses is the simultaneous periodicity in the beacon interval and the LTE-U duty cycle period. For instance, if the beacon interval is $102.4 \mathrm{~ms}$ with LTE-U ON and OFF periods as $6 \mathrm{~ms}$ and $4 \mathrm{~ms}$, respectively (i.e.,LTE-U ON fraction of 0.6); any beacon occurring at $0.8 \mathrm{~ms}$ from the start of LTE-U ON period would lead to a loss of 8 successive beacons. The obtained results were verified using MATLAB simulations. A slight mismatch in the experimental and simulated results is because of the fact that any extra beacons lost or received in the experiment apart from those in simulations, would result in generation of a new sequence of beacons lost.

Such beacon losses create the following problems.

1) Increased association delay: Since a victim user loses beacons transmitted by the AP, its association gets delayed. This delay is more pronounced with passive scanning where the Wi-Fi station has to wait for a beacon to get connected with the network [11]. When two or three beacons are lost in succession, the association delay will increase by the same multitude. However, the effect with active scanning is less serious unless if the probe response overlaps with the LTE-U ON period. In such cases another probe request needs to be transmitted. 
TABLE I

CONSECUTIVE BEACON LOSS (\%) OF VICTIM USERS FOR THE EXPERIMENTAL (EXPT) AND SIMULATION (SIMU) RESULTS.

\begin{tabular}{|c|c|c|c|c|c|c|}
\hline $\begin{array}{c}\text { No. of Consecutive } \\
\text { Beacon Losses }\end{array}$ & \multicolumn{2}{|c|}{$\begin{array}{c}\text { LTE-U ON } \\
\text { Fraction=0.2 }\end{array}$} & \multicolumn{2}{c|}{$\begin{array}{c}\text { LTE-U ON } \\
\text { Fraction=0.4 }\end{array}$} & \multicolumn{2}{c|}{$\begin{array}{c}\text { LTE-U ON } \\
\text { Fraction=0.6 }\end{array}$} \\
\hline- & Expt & Simu & Expt & Simu & Expt & Simu \\
\hline 1 & $\mathbf{3 0 . 8 4}$ & $\mathbf{3 3 . 6 5}$ & 3.65 & 0.47 & 0 & 0 \\
\hline 2 & $\mathbf{6 6 . 0 8}$ & $\mathbf{6 6 . 3 5}$ & $\mathbf{3 7 . 8 9}$ & $\mathbf{3 3 . 1 7}$ & 10.98 & 0 \\
\hline 3 & 1.76 & 0 & $\mathbf{5 3 . 8 8}$ & $\mathbf{6 6 . 3 5}$ & $\mathbf{7 4 . 0 5}$ & $\mathbf{8 0 . 0 0}$ \\
\hline 4 & 0.88 & 0 & 1.82 & 0 & 2.0 & 0 \\
\hline 8 & 0 & 0 & 0 & 0 & $\mathbf{1 1 . 3 9}$ & $\mathbf{1 9 . 4 3}$ \\
\hline
\end{tabular}

2) Increased disassociation frequency as a result of losing Channel Switch Information: Channel Switch Announcement (CSA) [11] is an important information which the Wi-Fi AP shares with its users before switching to a new channel. It sends out this information using beacons. If a user loses beacons containing CSA-in succession, then it may get disassociated and has to follow all the procedures again to re-associate itself.

3) Increased awake time and data latency for power-saving stations: Users with power saving mode enabled, wake up periodically at the correct beacon period and stay awake until they receive the beacon [11]. Transmission of beacons in an LTE-U ON period would cause the victim users to miss the beacon and remain awake for the entire beacon interval or some preset duration. Also, by losing beacons, these users would not be able to send PS-Poll frames (requesting the AP to transmit their data), thus increasing the delay in data received.

To mitigate the above effects, a quantification of beacon losses is necessary. Therefore, in the following section, we develop an analytical framework to determine the percentage of beacon losses and finally provide a mathematical expression for the same. This proposed expression also validates the results obtained using the testbed and simulations.

\section{BEACON LOSS ANALYSIS}

Let $B$ and $B_{\text {air-time }}$ be the beacon interval (defined as the duration between two successive beacons) and beacon airtime (defined as the duration required to transmit a complete beacon frame), respectively. Let $T$ denote the LTE-U duty cycle period with $T_{o n}$ and $T_{o f f}$ as the ON and OFF periods of the LTE-U. For finding the average beacon loss percentage, we define Beacon Start Time (BST) as the instant at which the beacon frame is transmitted with respect to the LTE-U duty cycle period. For example, $i_{1}$ in Fig. 9 denotes the first BST and $i_{2}$ denotes the second BST. Using these variables (i.e., $i_{1}, i_{2}, i_{3}, \ldots$ ), we calculate the beacon loss ratio and average it for all possible values of first BSTs. This gives the average beacon loss percentage. In addition, we make an assumption that the delay in beacon frames is negligible (i.e., the beacons arrive exactly at the start of beacon interval which is true for most of the cases).

If $i_{1}$ is the first BST, then the time at which the second beacon would arrive with respect to LTE-U duty cycle period will be $i_{2}=\left(i_{1}+B\right) \bmod T$, and similarly the third beacon

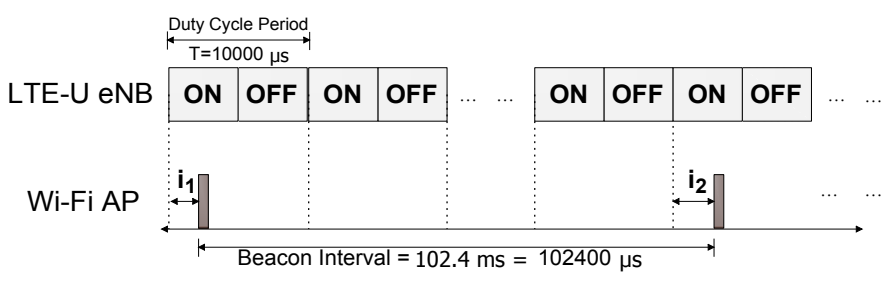

Fig. 9. Illustration of beacon arrival with respect to LTE-U duty cycle period.

would arrive at

$$
i_{3}=\left(\left(i_{1}+B\right) \bmod T+B\right) \bmod T=\left(i_{2}+B\right) \bmod T
$$

and hence the $n^{\text {th }}$ beacon arrival would arrive at

$$
i_{n}=\left(i_{n-1}+B\right) \bmod T
$$

For example, if $i_{1}$ is $10 \mu \mathrm{sec}$ in Fig. 9, $i_{2}$ and $i_{3}$ would be

$$
\begin{aligned}
& i_{2}=(10+102400) \bmod 10000=2410 \mu \mathrm{s} \\
& i_{3}=(2410+102400) \bmod 10000=4810 \mu \mathrm{s}
\end{aligned}
$$

Claim : The BST returns to the first BST $\left(i_{1}\right)$ after every $T$ beacon intervals, i.e., $i_{T+1}=i_{1}$.

Proof: We know that $i_{k}=\left(i_{k-1}+B\right) \bmod T$.

$$
\Longrightarrow i_{k}=\left(\left(i_{k-2}+B\right) \bmod T+B\right) \bmod T
$$

Using the addition property of modular arithmetic, we get

$$
\begin{gathered}
i_{k}=\left(i_{k-2} \bmod T+B \bmod T\right) \bmod T+B \bmod T \\
=\left(\left(i_{k-2} \bmod T+B \bmod T\right) \bmod T\right. \\
+B \bmod T) \bmod T
\end{gathered}
$$

Using the fact that $i_{k}$ 's are less than $T$, we get

$$
\begin{aligned}
i_{k} & =\left(\left(i_{k-2}+B \bmod T\right)+B \bmod T\right) \bmod T \\
& =\left(\left(i_{k-2}+2 \cdot B \bmod T\right)\right) \bmod T \\
& =\left(\left(i_{1}+(k-1) B\right) \bmod T\right) \bmod T
\end{aligned}
$$

Finally, $\quad i_{T+1}=\left(\left(i_{1}+T \cdot B\right) \bmod T\right) \bmod T$

Assuming $B \bmod T=k$, for some $k<T$

$$
\Longrightarrow i_{T+1}=\left(i_{1}+T \cdot k\right) \bmod T=i_{1}
$$

Now, we need to find the fraction of beacon losses, given the first BST was $i_{1}$. We know that, given $i_{1}$, the BSTs will follow a pattern as shown

$$
\left\{i_{1}, i_{2}, \ldots, i_{T-1}, i_{T}, i_{1}, i_{2}, \ldots, i_{T}, i_{1}, i_{2}, \ldots, i_{T}, i_{1}, \ldots\right\}
$$

As the duration of operation of Wi-Fi network tends to a large number, the pattern shown above repeats itself. Now, we need to use the fact that a beacon would be lost if its transmission overlaps with the LTE-U ON period (the ON period does not allow victim users to decode beacons successfully). This implies that if the BST occurs to be in one of the following two intervals, the beacons would be lost. Firstly, if the BST lies anywhere in the LTE-U ON period (i.e., $\left.\left(0, T_{\text {on }}\right)\right)$. Secondly, if the BST is in OFF period, but a part of beacon transmission 
overlaps with the upcoming ON period (due to the non-zero beacon air-time $\left.-B_{\text {air-time }}\right)$. Therefore, if the BSTs lie in $\left(0, T_{o n}\right)$ or $\left(T-B_{\text {air-time }}, T\right)$, the beacons can be considered as lost.

Now these BSTs from $i_{1}$ to $i_{T}$ can be divided into two sets, those lying in $\left(0, T_{o n}\right) \cup\left(T-B_{\text {air-time }}, T\right)$, called the lost set and others lying between $\left(T_{o n}, T-B_{\text {air-time }}\right)$, called the capture set. Let $m_{i}$ and $n_{i}$ denote the number of distinct BSTs from the set $\left\{i_{1}, i_{2}, \ldots, i_{T}\right\}$ belonging to the lost and capture sets, respectively. Note that, $m_{i}+n_{i}$ need not always be equal to $T$. In fact, many times the period of Eqn. (7) can be much smaller than $T$, however the upper bound is guaranteed to be $T$. Hence, the fraction of beacons lost with first BST as $i_{1}$ is

$$
L_{\text {frac }}\left(i_{1}\right)=\frac{m_{i}}{m_{i}+n_{i}}
$$

In addition, for first BSTs from $\left\{i_{2}, i_{3}, \ldots, i_{T}\right\}$, the set $\left\{i_{1}, i_{2}, \ldots, i_{T}\right\}$ will remain same, but the order in which the BSTs occur, would be slided. For example, if the first BST is $i_{3}$, the subsequent BSTs would follow a pattern as

$$
\left\{i_{3}, i_{4}, \ldots, i_{T}, i_{1}, i_{2}, i_{3}, \ldots, i_{T}, i_{1}, i_{2}, \ldots, i_{T}, i_{1}, i_{2}, \ldots\right\}
$$

This would imply that $m_{i}$ and $n_{i}$ would remain same, and consequently $L_{\text {frac }}$ would also remain same.

Therefore, $\quad L_{\text {frac }}\left(i_{1}\right)=L_{\text {frac }}\left(i_{2}\right)=\ldots=L_{\text {frac }}\left(i_{T}\right)$

Now, consider any other first BST $j_{1}$, such that $j_{1} \notin$ $\left\{i_{1}, i_{2}, \ldots, i_{T}\right\}$. This would imply that $j_{1}$ would produce a new set $\left\{j_{1}, j_{2}, \ldots, j_{T}\right\}$ with new $m_{j}$ and $n_{j}$. Consequently, the beacon loss fraction for these BSTs would be

$$
L_{\text {frac }}\left(j_{1}\right)=L_{\text {frac }}\left(j_{2}\right)=\ldots=L_{\text {frac }}\left(j_{T}\right)=\frac{m_{j}}{m_{j}+n_{j}}
$$

Similarly, consider any other first BST $k_{1}$, such that $k_{1} \notin$ $\left\{i_{1}, i_{2}, \ldots i_{T}\right\} \cup\left\{j_{1}, j_{2}, \ldots j_{T}\right\}$. This would again produce $m_{k}$ and $n_{k}$ with the beacon loss fraction to be

$L_{\text {frac }}\left(k_{1}\right)=L_{\text {frac }}\left(k_{2}\right)=\ldots=L_{\text {frac }}\left(k_{T}\right)=\frac{m_{k}}{m_{k}+n_{k}}$

After exhausting the complete duty cycle period $T$, the average beacon loss fraction can be computed as

$$
L_{\text {frac }}=\frac{\sum_{i=1}^{T} L_{\text {frac }}(i)}{T}=\frac{\sum_{p=\{i, j, k, \ldots\}} \sum_{x=1}^{m_{p}+n_{p}} L_{f r a c}\left(p_{x}\right)}{T}
$$

Since $L_{\text {frac }}\left(p_{x}\right)$ is constant for all $x \in$ either $\left\{i_{1}, i_{2}, \ldots, i_{T}\right\}$, or $\left\{j_{1}, j_{2}, \ldots, j_{T}\right\}$ and so on, $L_{\text {frac }}$ will reduce to

$$
L_{\text {frac }}=\frac{\sum_{p=\{i, j, k, \ldots\}} \frac{m_{p}}{m_{p}+n_{p}} \cdot\left(m_{p}+n_{p}\right)}{T}=\frac{\sum_{p=\{i, j, k, \ldots\}} m_{p}}{T}
$$

Since, the complete duration from $(0, T]$ was exhausted by selecting appropriate first BSTs, the sum of all $m_{p^{\prime} s}$ should be equal to the size of lost set.

$$
\Longrightarrow \sum_{p=\{i, j, k, \ldots\}} m_{p}=T_{\text {on }}+B_{\text {air-time }}
$$

Therefore, the average beacon loss fraction is given by

$$
L_{\text {frac }}=\frac{T_{\text {on }}+B_{\text {air-time }}}{T}
$$

For a special case when $T_{o n}>T-B_{\text {air-time }}$, the LTE-U OFF period would be insufficient for the victim users to receive any beacon successfully, making the lost set as $(0, T)$. Similarly, when $T_{o n}=0$, the scenario reduces to a simple only Wi-Fi scenario and thus the lost set would be a null set $(\phi)$. This implies the expression for $L_{\text {frac }}$ considering all the conditions would be

$$
L_{\text {frac }}= \begin{cases}0 & \text { if } T_{\text {on }}=0 \\ 1 & \text { if } T_{\text {on }}>T-B_{\text {air-time }} \\ \frac{T_{\text {on }}+B_{\text {air-time }}}{T} & \text { otherwise }\end{cases}
$$

The above beacon loss analysis is validated using the testbed described in Section III and also using MATLAB simulations. For both the testbed and simulations the beacon interval was set to $102.4 \mathrm{~ms}$. In general, AP uses the lowest rate to transmit beacons, hence the beacon rate in our setup was observed to be $1 \mathrm{Mb} / \mathrm{s}$ with a beacon size of $287 \mathrm{~B}$. As a result, the beacon air-time of $2.3 \mathrm{~ms}$ was used in the simulations and analysis.

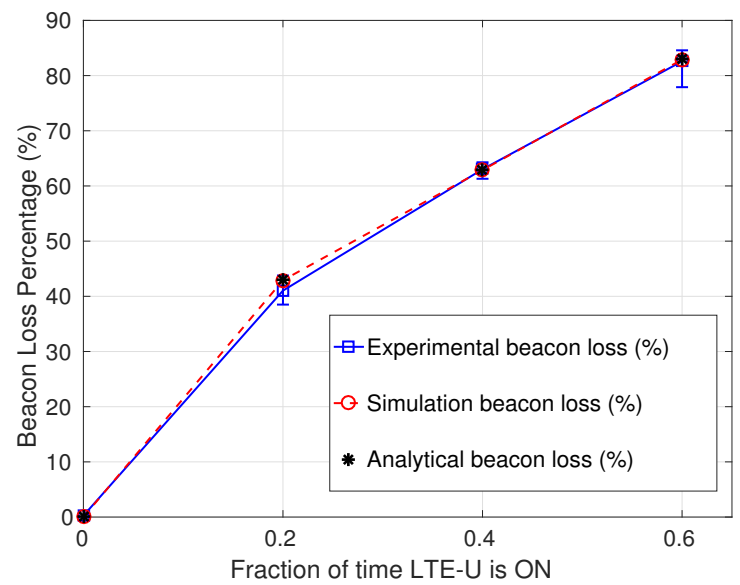

Fig. 10. Validation of analytical beacon loss percentage (\%) of the victim user through testbed experiment and simulation results for an LTE-U duty cycle period of $10 \mathrm{~ms}$ with varying $\mathrm{ON}$ fraction.

Fig. 10 validates the beacon loss percentage results collected for a duty cycle period of $10 \mathrm{~ms}$ with different LTE-U ON fractions. The simulation and analysis curves match closely with the testbed results, thus confirming their correctness. Moreover, it also shows an increase in beacon loss percentage of victim user with LTE-U ON period. This comes from the fact that the average beacon loss fraction $\left(L_{\text {frac }}\right)$ is indeed a 
linear function of LTE-U ON fraction, and can be obtained by simplifying Eqn. (16) as

$$
L_{f r a c}=\text { LTE-U_ON_Fraction }+\frac{B_{\text {air }- \text { time }}}{T}
$$

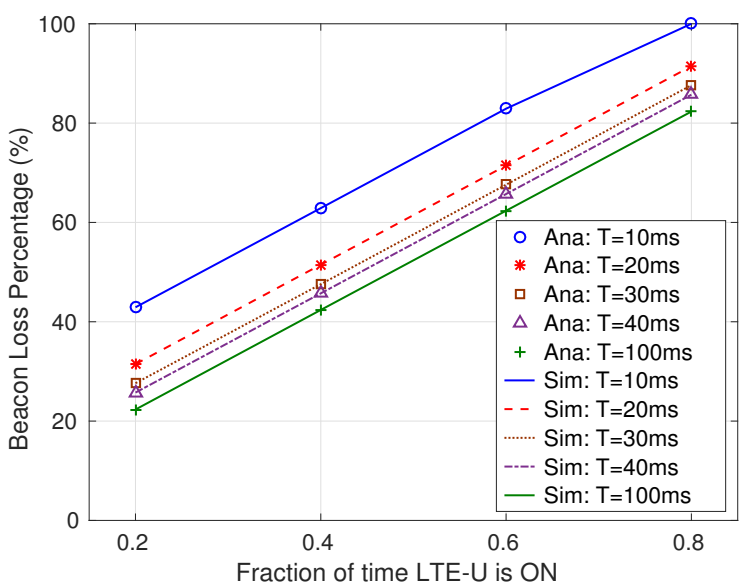

Fig. 11. Analytical (Ana) and Simulated (Sim) beacon loss percentage (\%) of victim user for different LTE-U duty cycle periods with varying ON fraction.

Fig. 11 shows the variation in beacon loss percentage with increasing LTE-U duty cycle period. It shows that the beacon loss percentage decreases with increasing LTE-U duty cycle period, but finally saturates near the LTE-U ON percentage (for very high duty cycle periods), with the second term in Eqn. (17) becoming negligible. However, an important consideration before increasing LTE-U duty cycle period is that higher periods become a bottleneck for satisfying Quality of Service (QoS) requirement of the Wi-Fi network.

\section{CONClusions AND Future WORK}

In this paper, we have shown the impact of duty cycled LTE-U on the performance of Wi-Fi users in the hidden terminal scenario, using testbed experiments. The results demonstrate a surprising behavior, with the fairness among $\mathrm{Wi}-\mathrm{Fi}$ users completely forfeited and a declination in the network throughput as well. In fact, the users in the considered scenario were divided into two groups, with one group, apart from receiving lower throughput, was also deprived from listening to periodic beacons. These beacon losses were quantified by performing a testbed experiment and then was throughly validated using simulations and mathematical analysis. In addition, issues related to successive beacon losses like delay in association, frequent disassociation, etc were also highlighted. Consequently, the paper shows that the channel access schemes for LTE in unlicensed, like duty cycled LTE-U or LBT based LAA need additional functionality to address these hidden terminal problems.

Although hidden terminal problem has been well studied for the Wi-Fi deployments, the presence of a different Radio Access Technology (RAT) utilizing the same unlicensed spectrum, i.e., an LTE-U/LAA, makes this problem challenging.
It requires a need to look into the complication created by presence of the LTE-U, which we delineated extensively in our work, so as to find a solution. Our analysis makes us believe that an elementary step towards this problem would be to employ the existing Request-To-Send (RTS) and Clear-ToSend (CTS) or Self-CTS mechanisms, which a Wi-Fi network extensively uses, within the competing RAT as well, with necessary changes. Hence, as a part of future work we intend to solve this issue to ensure a better and fair coexistence of LTE-U and Wi-Fi in the unlicensed spectrum.

\section{ACKNOWLEDGMENTS}

This work was supported by the project "Converged Cloud Communication Technologies", Meity, Govt. of India.

\section{REFERENCES}

[1] Ericsson, "Ericsson mobility report," June 2017.

[2] C. W. Paper, "Cisco visual networking index: global mobile data traffic forecast update, 2016-2021," Feb 2017.

[3] 3GPP, "TSGRAN; Study on Licensed-Assisted Access to Unlicensed Spectrum,” Tech. Rep. TR 36.889 V13.0.0, June 2015.

[4] "LTE-U SDL CSAT Procedure Technical Specifications V1.0.” LTE-U Forum, 2015.

[5] LTE-U Forum, "LTE-U Forum online." [Online] http://www.lteuforum. org, 2015.

[6] LTE-U Forum, "LTE-U Technical Report." [Online] http://www. lteuforum.org/documents.html, 2015.

[7] LTE-U Forum, "UE Minimum Requirements for LTE-U SDL," 2015.

[8] LTE-U Forum, "eNB Minimum Requirements for LTE-U SDL." [Online] http://www.lteuforum.org/documents.html, 2015.

[9] "LTE in Unlicensed Spectrum: Harmonious Coexistence with Wi-Fi." Qualcomm White Paper, June 2014.

[10] C. Cano and D. J. Leith, "Unlicensed LTE/WiFi Coexistence: Is LBT Inherently Fairer Than CSAT?," in IEEE International Conference on Communications (ICC), July 2016.

[11] I. S. Association et al., "802.11-2012-IEEE Standard for Information technology-Telecommunications and information exchange between systems Local and metropolitan area networks-Specific requirements Part 11: Wireless LAN Medium Access Control (MAC) and Physical Layer (PHY) Specifications," Retrived from http://standards. ieee. org/about/get/802/802.11. html, 2012.

[12] F. M. Abinader, E. P. Almeida, F. S. Chaves, A. M. Cavalcante, R. D. Vieira, R. C. Paiva, A. M. Sobrinho, S. Choudhury, E. Tuomaala, K. Doppler, et al., "Enabling the coexistence of LTE and Wi-Fi in unlicensed bands," IEEE Communications Magazine, vol. 52, no. 11, pp. 54-61, 2014.

[13] A. Babaei, J. Andreoli-Fang, Y. Pang, and B. Hamzeh, "On the impact of LTE-U on Wi-Fi performance," International Journal of Wireless Information Networks, vol. 22, no. 4, pp. 336-344, 2015.

[14] A. M. Cavalcante, E. Almeida, et al., "Performance evaluation of LTE and Wi-Fi coexistence in unlicensed bands," in IEEE 77th Vehicular Technology Conference (VTC Spring), 2013.

[15] T. Nihtilä, V. Tykhomyrov, O. Alanen, et al., "System performance of LTE and IEEE 802.11 coexisting on a shared frequency band," in IEEE Wireless Communications and Networking Conference (WCNC), 2013.

[16] E. Almeida, A. M. Cavalcante, et al., "Enabling lte/wifi coexistence by lte blank subframe allocation," in IEEE International Conference on Communications (ICC), 2013.

[17] V. Maglogiannis, D. Naudts, et al., "Impact of LTE Operating in Unlicensed Spectrum on Wi-Fi Using Real Equipment," in IEEE Global Communications Conference (GLOBECOM), 2016.

[18] "Real-time LTE/Wi-Fi Coexistence Testbed." National Instruments White Paper, http://www.ni.com/white-paper/52119/en/, Feb 2016.

[19] "LabVIEW Communications LTE Application Framework 1.1." National Instruments, http://www.ni.com/white-paper/52524/en/, Mar 2015.

[20] "iPerf." Jon Dugan, Seth Elliott, Bruce A. Mah, Jeff Poskanzer, Kaustubh Prabhu, [online] https://iperf.fr/, accessed Mar 2017.

[21] Wireshark. [online] https://www.wireshark.org, accessed on March 2017. 